\title{
A New Hyperprior Distribution for Bayesian Regression Model with Application in Genomics
}

\author{
Renato Rodrigues Silva ${ }^{1, *}$ \\ 1 Institute of Mathematics and Statistics, Federal University of Goiás, \\ Goiânia, Goiás, Brazil \\ *renato.rrsilva@ufg.br
}

\begin{abstract}
In the regression analysis, there are situations where the model have more predictor variables than observations of dependent variable, resulting in the problem known as "large p small n". In the last fifteen years, this problem has been received a lot of attention, specially in the genome-wide context. Here we purposed the bayes $\mathrm{H}$ model, a bayesian regression model using mixture of two scaled inverse chi square as hyperprior distribution of variance for each regression coefficient. This model is implemented in the $\mathrm{R}$ package BayesH.
\end{abstract}

\section{Introduction}

In the regression analysis, there are situations where the model have more predictor variables than observations of dependent variable, resulting in the problem known as "large p small n" [1].

To figure out this problem, there are already exists some methods developed as ridge regression [2], least absolute shrinkage and selection operator (LASSO) regression [3], bridge regression [4], smoothly clipped absolute deviation (SCAD) regression 5] and others. This class of regression models is known in the literature as regression model with penalized likelihood [6. In the bayesian paradigma, there are also some methods purposed as stochastic search variable selection [7], and Bayesian LASSO [8].

Recently, the "large p small n" problem has been receiving more attention for scientist who works with animal or plant genetics, specifically to apply in genome-wide selection studies 9].

Genome-wide selection is a approach in quantitative genetics to predict the breeding value of the individuals from a testing population based on estimates of the molecular marker effects from training population. The training population is comprised by individuals which were genotyped and phenotyped while in the testing population the individuals are only genotyped [10, [11]. Genotyping refers to obtain the genetic makeup of individuals through some technology 12 and phenotyping is a measure of some economic importance traits as yield, height and etc 11,13.

With advent of the high throughput genotyping plataforms, nowadays is possible to define a statistical model to identify association between molecular markers and an observed phenotype. In these models, the effects of all markers are estimated simultaneously, capturing even small effects 10,14.

In the context of genome-wide selection, many animal and plant breeders developed some bayesian regression models to make prediction of complex traits when there are 
more covariables than observations of response variable. In the cornerstone publication [10], Bayes A and Bayes B models were presented. In the Bayes A, the scaled-t density were used as prior distribution of marker effects, while in the Bayes B the prior distribution were modeled using a mixture of a point of mass at zero and a scaled-t density. More recently, the use of a mixture of a point of mass at zero and a Gaussian slab were purposed. This model is known in the literature as Bayes $\mathrm{C} \pi$ 15, 17,19 .

However, there are issues in these models which should have been taken into account. The prior distribution is always influential, therefore its choice is crucial. In this paper we proposed the fit of an Bayesian regression model with mixture of two scaled inverse chi square as hyperprior distribution of variance for each regression coefficient (bayes $\mathrm{H}$ model). Until our knowledge, it has never reported before.

An advantage of the model is the flexibility. Depending on values chosen for hyperparameters, is possible to obtain equivalent models to (Bayes Ridge Regression and Bayes A) or even to select variable via Gibbs Sampling in a broad sense. To illustrate to application of the Bayes $\mathrm{H}$ model, we analyzed some simulated and real datasets.

\section{Materials and Methods}

\section{Simulated Data}

The aim these simulations were compare effects of prior distribution in the prediction of complex traits in some situations such as presence or absence of strong linkage disequilibrium or oligogenic or poligenic genetic architecture. The parameter settings of four scenarios generated are presented below. The phenotype were calculated using the equation described by (2).

Table 1. Parameter settings for four simulated scenarios

\begin{tabular}{cccccc}
\hline Scenario & Population $*$ & N. individuals & N. markers & N. QTL's & Distribution of QTL's effect \\
\hline 1 & heterogeneous stock mice & 250 & 2500 & 50 & Gamma $(3 ; 0,75)$ \\
\hline 2 & heterogeneous stock mice & 250 & 2500 & 10 & Gamma(3;0,75) \\
\hline 3 & random mating & 250 & 2500 & 50 & Gamma(3;0,75) \\
\hline 4 & random mating & 250 & 2500 & 10 & Gamma $(3 ; 0,75)$ \\
\hline
\end{tabular}

* Heterogeneous stock mice were sampled from subsampling of mice dataset , which had already analized by [25] and avalaible in BGLR library of R statistical software [19]; random mating were sampled from Bernoulli distribution with allele frequency equal to 0.5 .

For each scenario the predictive performance between Bayes ridge regression and Bayes H model were compared. The table (1) displays the values of hyperparameters used in mixture of the scaled inverse chi-squared distribution. Depending on the values assigned to hyperparameters, differents model can be defined. For example, using the hyperprior A (1), the model will be equivalent to Bayes A model [10]. On the other hand, the use of hyperprior B can be considered as a variable selecion model in a broad sense.

Table 2. Information about hyperparameters used in each component of mixture

\begin{tabular}{crcrc}
\hline Hyperprior & $\nu_{1}$ & $s_{1}$ & $\nu_{2}$ & $s_{2}$ \\
\hline $\mathrm{A}$ & 5 & 0.04 & 5 & 0.04 \\
\hline $\mathrm{B}$ & 5 & 0.5 & 7 & 0.002 \\
\hline
\end{tabular}

The values of hyperparameters for prior distribuition for $\sigma^{2}$ were defined as follows: degree of freedom equal to 5 and scale parameter equal to 0.1 . Figure (1) shows the 
influence of hyperprior distribution for $\tau^{2}$ in the marginal prior for $\beta_{j}$. Assuming $\sigma^{2}=1$, it is observed the use of hyperprior A (mixture with same scale parameters) the marginal prior resulting for $\beta_{j}$ is a t-scaled distribution. On the contrary, when hyperprior $\mathrm{B}$ is used the marginal prior obtaining for $\beta_{j}$ is a mixture of t-scaled distribution with the same location parameters but different scales parameters, which results a distribution with tails heavier and sharper peaks than t-scaled.

Fig 1. Hyperprior distribution for $\tau^{2}$ and marginal prior distribution for $\beta_{j}$

\section{Real Data}

The real dataset is comprised by 10346 polymorphic markers scored in a mice population of 1814 individuals. The phenotype measured was body mass index (BMI) 28]. The dataset were previously by [25], which further details about about heterogeneous stock mice population can be found. It is important to mention the dataset is avalaible in R package BGLR 19 .

Predictions of the complex traits in the mice dataset was done in two step. First of all, a mixed model was fitted to remove the population structure and kinship effect of dataset. In the second step, Bayes $\mathrm{H}$ or Bayesian Ridge Regression were fitted to make predictions considering the BLUP's predicted from mixed model as response variable.

The inference of population was based on clustering of the loadings of two top principal components obtained from to genomic relationship matrix. The clustering was done using Gaussian mixture models implemented in the library Mclust of $\mathrm{R}$ statistical software [23], 29] e 30. Several mixture models were fitted and bayesian information criterion was used to select the best model. The candidate models differ each other in relation to covariance matrix of each component of mixture. The general structure of covariance matrix is $\Sigma_{k}=\lambda D_{k} A_{k} D_{k}^{\prime}$ where $\Sigma_{k}$ is the covariance matrix of $k t h$ component of mixture model, $D_{k}$ is the orthogonal matrix of eigenvectors, $A_{k}$ is the diagonal matrix whose elements are proportional to the eigenvalues and $\lambda$ is a scale parameter 30].

\section{Phenotypic Analysis - Mixed Model}

Before to predict the molecular breeding value of BMI the phenotypic analysis was done. Phenotypic analysis consisted fitting the mixed model to heterogeneous stock mice population and predict the best linear unbiased predictor (BLUP) for each individual 10,14 .

The mixed model is defined by

$$
\boldsymbol{y}=\mathbf{X} \boldsymbol{\beta}+\mathbf{Z b}+\boldsymbol{\epsilon}
$$

where $\boldsymbol{y}$ is the vector of response variable; $\mathbf{X}$ is the incidence matrix of the fixed effects; $\boldsymbol{\beta}$ is the vector of fixed effects that represents (litter, gender, year and population strucuture); $\mathbf{Z}$ the incidence matrix of random effects; $\mathbf{b}$ the vector of random effects that follows Gaussian distribution with mean 0 and variance $\sigma_{b}^{2}$ and $\epsilon$ the random error.

Population structure was infered using the results from fitting of Gaussian Mixture Models implemented in the package mclust, a library of $\mathrm{R}$ statistical software $23,29,30$. 


\section{Genomic Selection - Statistical Model}

The statistical model is defined by

$$
y_{i}=\mu+\sum_{k=1}^{p} \beta_{k} x_{i k}+\epsilon_{i}
$$

where $y_{i}$ is the $i-t h$ observation of response variable; $\beta_{k}$ is the $k-t h$ regression coefficient of model; $x_{i k}$ is a explanatory variable for $i-t h$ individual and $k-t h$ explanatory variable and $\epsilon_{i}$ is the random error for $i-t h$ individual that follows $N\left(0, \sigma^{2}\right)$.

\section{Prior Distributions}

Considering the fitting of the model, the prior distribution for intercept $\mu$, is defined by

$$
\mu \sim N\left(0, \omega^{2}\right)
$$

where $\omega^{2}$ is a hyperparameter. In practice, we used a large number for $\omega^{2}$ to set up this prior distribution as vague.

The prior distribution for each $\beta_{k}$ given a value of $\tau_{k}^{2}$ is Gaussian, i.e,

$$
\beta_{k} \mid \tau_{k}^{2}, \sigma^{2} \sim N\left(0, \tau_{k}^{2} \sigma^{2}\right)
$$

Here, is the novelty of the manuscript. The hyperprior distribution for $\tau_{k}^{2}$ is conditioned a latent random variable $Z_{k}$. Hence, the hyperprior distribution for $\tau_{k}^{2}$ follows the mixture of the two components scaled inverse chi square distribution, i.e

$$
\left\{\begin{array}{l}
Z_{k} \sim \text { Bernoulli }(1, \pi) \\
\tau_{k}^{2} \mid Z_{k}=1, \sim \text { Scaled-Inv } \chi^{2}\left(\nu_{1}, s_{1}^{2}\right) \\
\tau_{k}^{2} \mid Z_{k}=0, \sim \text { Scaled-Inv } \chi^{2}\left(\nu_{2}, s_{2}^{2}\right)
\end{array}\right.
$$

The hyperprior distribution of $\pi$ is Beta distribution with parameters $(\alpha, \gamma)$. In pratice, we adopted $\alpha=1$ and $\gamma=1$ to obtain a vague hyperprior.

Finally, the prior distribution for $\sigma$ is

$$
\sigma^{2} \sim \operatorname{Scaled}-\operatorname{Inv} \chi^{2}\left(\nu_{\sigma}, s_{\sigma}^{2}\right)
$$

where the hyperparameters $\nu_{\sigma}, s_{\sigma}^{2}$ represents the degree of freedom and scale parameters of the scaled inverse chi-square distribution.

\section{Likelihood and Posterior Distribution}

The likelihood is defined by

$$
\operatorname{Pr}\left(\mathbf{y}, \mu, \boldsymbol{\beta}, \tau^{2}, \sigma^{2}\right)=\frac{1}{\left(\sigma^{2}\right)^{\frac{n}{2}}} \exp \left\{-\frac{1}{2 \sigma^{2}} \sum_{i=1}^{n}\left(y_{i}-\mu-\sum_{k=1}^{p} \beta_{k} x_{i k}\right)^{2}\right\}
$$

Hence, the joint posterior distribution is given by

$$
\begin{aligned}
\operatorname{Pr}\left(\mu, \boldsymbol{\beta}, \tau_{k}^{2}, \sigma^{2} \mathbf{z}, \pi \mid \mathbf{y}\right) \propto & \operatorname{Pr}\left(\mathbf{y} \mid \mu, \boldsymbol{\beta}, \sigma^{2}\right) \operatorname{Pr}(\mu) \prod_{k=1}^{p}\left[\operatorname{Pr}\left(\beta_{k} \mid \tau_{k}^{2}\right) \operatorname{Pr}\left(\tau_{k}^{2} \mid Z_{k}\right) \operatorname{Pr}\left(Z_{k} \mid \pi\right)\right] \operatorname{Pr}(\pi) \times \\
& \operatorname{Pr}\left(\sigma^{2}\right)
\end{aligned}
$$




$$
\begin{aligned}
& \operatorname{Pr}\left(\mu, \boldsymbol{\beta}, \tau^{2}, \sigma^{2} \mathbf{z}, \pi \mid \mathbf{y}\right) \propto \frac{1}{\left(\sigma^{2}\right)^{\frac{n}{2}}} \exp \left\{-\frac{1}{2 \sigma^{2}} \sum_{i=1}^{n}\left(y_{i}-\mu-\sum_{k=1}^{p} \beta_{k} x_{i k}\right)^{2}\right\} \\
& \exp \left\{-\frac{1}{2 \omega^{2}} \mu^{2}\right\} \prod_{k=1}^{p}\left[\left(\pi \frac{1}{\tau_{k}^{2\left(\frac{\nu_{1}}{2}+1\right)}} \exp \left\{-\frac{\nu_{1} s_{1}^{2}}{2 \tau^{2}}\right\}\right)^{I\left(Z_{k}=1\right)}\right. \\
& \left.\left((1-\pi) \frac{1}{\tau_{k}^{2\left(\frac{\nu_{2}}{2}+1\right)}} \exp \left\{-\frac{\nu_{2} s_{2}^{2}}{2 \tau_{k}^{2}}\right\}\right)^{I\left(Z_{k}=0\right)} \frac{1}{\left(\tau_{k}^{2}\right)^{\frac{1}{2}}} \exp \left\{-\frac{1}{2 \tau_{k}^{2}} \beta_{k}^{2}\right\}\right] \\
& \frac{1}{B(\alpha, \gamma)} \pi^{\alpha-1}(1-\pi)^{\gamma-1} \frac{1}{\left(\sigma^{2}\right)^{\left(\frac{\nu_{\sigma}}{2}+1\right)}} \exp \left\{-\frac{\nu_{\sigma} s_{\sigma}^{2}}{2 \sigma^{2}}\right\}
\end{aligned}
$$

\section{Gibbs sampling algorithm}

Gibbs sampling was used to obtain a sequence of observed values of the parameters 20], 21. The full conditional posterior distribution for $\mu$ is given by

$$
\mu^{(g)} \mid \boldsymbol{\beta}^{(g-1)}, \tau_{k}^{2(g-1)}, \sigma^{2(g-1)}, \pi^{(g-1)}, \mathbf{z}^{(g-1)}, \mathbf{y} \sim N\left(\tilde{\mu}^{(g)}, \tilde{\sigma}^{2(g)}\right)
$$

where

$$
\begin{gathered}
\tilde{\mu}^{(g)}=\frac{\frac{\sum_{i=1} y_{i}^{*(g)}}{\sigma^{2(g-1)}}}{\left(\frac{n}{\sigma^{2(g-1)}}+\frac{1}{\omega^{2(g-1)}}\right)} \\
\tilde{\sigma}^{2(g)}=\frac{1}{\left(\frac{n}{\sigma^{2(g-1)}}+\frac{1}{\omega^{2(g-1)}}\right)} \\
y_{i}^{*(g)}=y_{i}-\sum_{k=1}^{p} \beta_{k}^{(g-1)} x_{i k}
\end{gathered}
$$

and $g$ is the counter of Gibbs sampling algorithm.

The full conditional posterior distribution for $\tau_{k}^{2}$ given $Z_{k}=1$ is defined by

$$
\tau_{k}^{2(g)} \mid \mu^{(g)}, \boldsymbol{\beta}^{(g-1)}, \pi^{(g-1)}, \sigma^{2(g-1)}, Z_{k}=1, \mathbf{y} \sim \operatorname{Scaled-Inv} \chi^{2}\left(\nu_{1}+1, \frac{\frac{\beta_{k}^{2(g-1)}}{\sigma^{2(g-1)}}+\nu_{1} s_{1}^{2}}{\nu_{1}+1}\right)
$$

Likewise, for given $Z_{k}=0$ we have

$$
\tau_{k}^{2(g)} \mid \mu^{(g)}, \boldsymbol{\beta}^{(g-1)}, \pi^{(g-1)}, \sigma^{2(g-1)}, Z_{k}=0, \mathbf{y} \sim \text { Scaled-Inv } \chi^{2}\left(\nu_{2}+1, \frac{\frac{\beta_{k}^{2(g-1)}}{\sigma^{2(g-1)}}+\nu_{2} s_{2}^{2}}{\nu_{2}+1}\right)
$$

The values of $z_{k}$ are obtained computing the probability $Z_{k}$ given $\tau_{k}^{(2 g)}$ and $\pi^{(g)}$, i.e, ${ }^{123}$

$$
\operatorname{Pr}\left(Z_{k}=1 \mid \tau_{k}^{2(g)}, \pi^{(g-1)}\right)=\frac{\pi f_{1}\left(\tau_{k}^{2}\right)}{\pi f_{1}\left(\tau_{k}^{2}\right)+(1-\pi) f_{2}\left(\tau_{k}^{2}\right)}
$$

where $f_{1}\left(\tau^{2}\right) f_{2}\left(\tau^{2}\right)$ are probability density functions of scaled inverse chi square distribution with parameters $\left(\nu_{1}, s_{1}^{2}\right)$ and $\left(\nu_{2}, s_{2}^{2}\right)$, respectively. 
Moreover,

$$
Z_{k} \sim \operatorname{Bernoulli}\left(1, \operatorname{Pr}\left(Z_{k}=1 \mid \tau_{k}^{2(g)}, \pi^{(g-1)}\right)\right)
$$

The full conditional posterior distribution for $\pi$ is defined by

$$
\pi^{(g)} \mid \mu^{(g)}, \boldsymbol{\beta}^{(g-1)}, \tau_{k}^{2(g)}, \sigma^{2(g-1)}, \mathbf{z}^{(g)}, \mathbf{y} \sim \operatorname{Beta}\left(\alpha+\sum_{z_{k}: z_{k}=1} z_{k}^{(g)}, \gamma+p-\sum_{z_{k}: z_{k}=1} z_{k}^{(g)}\right)
$$

The procedure to sampling $\beta_{k}$ given $Z_{k}=1$ from the full conditional posterior distribution was adapted from the strategy purposed by 22], i.e

where

$$
\beta_{k}^{(g)} \mid \mu^{(g)}, \tau_{k}^{2(g)}, \sigma^{2(g-1)}, \pi^{g)}, \boldsymbol{\beta}_{-k}^{(g-1)}, Z_{k}=1, \mathbf{y} \sim N\left(\dot{\beta}_{k}^{(g)}, \dot{\sigma}^{2(g)}\right)
$$

$$
\begin{gathered}
\dot{\beta}_{k}^{(g)}=\frac{\frac{\sum_{i=1} x_{i j} y_{i k}^{* *(g)}}{\sigma^{2(g-1)}}}{\frac{\sum_{i=1} x_{i j}^{2}}{\sigma^{2(g-1)}}+\frac{1}{\sigma^{2(g-1)} \tau_{k}^{2(g)}}} ; \\
\dot{\sigma}_{k}^{2(g)}=\frac{1}{\frac{\sum_{i=1} x_{i j}^{2}}{\sigma^{2(g-1)}}+\frac{1}{\sigma^{2(g-1)} \tau_{k}^{2(g)}}} ;
\end{gathered}
$$

and

$$
y_{i k}^{* *(g)}=y_{i}-\sum_{j \neq k} \beta_{j}^{(g-1)} x_{i j} .
$$

Finally, the full conditional posterior distribution for $\sigma^{2}$ is given by

$$
\sigma^{2(g)} \mid \mu^{(g)}, \boldsymbol{\beta}^{(g)}, \tau_{k}^{2(g)}, \mathbf{y} \sim \text { Scaled-Inv } \chi^{2}\left(\nu_{\sigma}+n, \frac{\mathrm{SSE}+\mathrm{SSB}+\nu_{\sigma} s_{\sigma}^{2}}{\nu_{\sigma}+n+p}\right)
$$

where $\mathrm{SSE}=\sum_{i=1}^{n}\left(y_{i}-\mu-\sum_{k=1}^{p} \beta_{k}^{(g)} x_{i k}\right)^{2}$ and $\mathrm{SSB}=\sum_{k=1}^{p} \frac{\beta_{k}^{2}}{\tau_{k}^{2}}$.

For Bayesian Ridge Regression, there are a unique $\tau^{2}$ hyperparameter. The prior distribution for $\tau^{2}$ and $\sigma^{2}$ follows scaled inverse chi square with hyperparameters $\left(\nu, s^{2}\right)$ and $\left(\nu_{\sigma}, s_{\sigma}^{2}\right)$.

The full conditional posterior distribution for $\tau^{2}$ is

$$
\tau^{2(g)} \mid \mu^{(g)}, \boldsymbol{\beta}^{(g-1)}, \pi^{(g-1)}, \sigma^{2(g-1)}, \mathbf{y} \sim \operatorname{Scaled-Inv} \chi^{2}\left(\nu+p, \frac{\frac{\sum_{k=1}^{p} \beta_{k}^{(2(g-1)}}{\sigma^{2(g-1)}}+\nu s^{2}}{\nu+p}\right)
$$

and for $\sigma^{2}$, we have

$$
\sigma^{2(g)} \mid \mu^{(g)}, \boldsymbol{\beta}^{(g)}, \tau^{2(g)}, \mathbf{y} \sim \text { Scaled-Inv } \chi^{2}\left(\nu_{\sigma}+n+p, \frac{\mathrm{SSE}+\mathrm{SSB}+\nu_{\sigma} s_{\sigma}^{2}}{\nu_{\sigma}+n+p}\right)
$$

Consequently, the full conditional posterior for $\beta_{k}$ parameters is given by

$$
\beta_{k}^{(g)} \mid \mu^{(g)}, \tau^{2(g-1)}, \sigma^{2(g-1)}, \pi^{(g-1)}, \boldsymbol{\beta}_{-k}^{(g-1)}, Z_{k}=1, \mathbf{y} \sim N\left(\dot{\beta}_{k}^{(g)}, \dot{\sigma}^{2(g)}\right)
$$


where

$$
\begin{gathered}
\dot{\beta}_{k}^{(g)}=\frac{\frac{\sum_{i=1} x_{i j} y_{i k}^{* *(g)}}{\sigma^{2(g-1)}}}{\frac{\sum_{i=1} x_{i j}^{2}}{\sigma^{2(g-1)}}+\frac{1}{\sigma^{2(g-1)} \tau^{2(g)}}} \\
{\dot{\sigma^{2}}}_{k}^{(g)}=\frac{1}{\frac{\sum_{i=1} x_{i j}^{2}}{\sigma^{2(g-1)}}+\frac{1}{\sigma^{2(g-1)} \tau^{2(g)}}} .
\end{gathered}
$$

In summary, the Gibbs sampling algorithm can be defined by

For 1 to $\mathrm{G}$ do it:

1- Generate $\mu^{(g)}$ from 8 .

2- Generate $\tau^{2(g)}$ from $(9)$.

3- Generate $\mathbf{z}^{(g)}$ from 11 and 12 .

4- Generate $\pi^{(g)}$ from 13 .

5 - Generate each $\beta_{k}^{(g)}$ from 14 .

6- Generate $\sigma^{2(g)}$ from 15 .

where $G$ is the number of iterations.

This algorithm was implemented in a $\mathrm{R}$ package 23 called BayesH avalaible at https://cran.r-project.org/web/packages/BayesH/index.html.

\section{Mathematical Details about Prior Distribution}

In this section we are going to show some details about conditional prior distribution for $\beta_{k} \mid \tau_{k}^{2}$ given a prior distribuition for $\tau_{k}^{2} \mid Z_{k}$. This demonstration is based on 17. The distribution of hyperparameter $\tau_{k}^{2}$ for given $Z_{k}=1$ is described by

$$
f_{\tau_{k}^{2} \mid z_{k}=1}\left(\tau_{k}^{2}\right)=\frac{\left(\frac{s_{1}^{2} \nu_{1}}{2}\right)^{\frac{\nu_{1}}{2}}}{\Gamma\left(\frac{\nu_{1}}{2}\right)} \exp \left\{-\frac{1}{2}\left[\frac{s_{1}^{2} \nu_{1}}{\tau_{k}^{2}}\right]\right\} \frac{1}{\tau_{k}^{2}\left(1+\frac{\nu_{1}}{2}\right)},
$$

and the prior distribution for $\beta_{k}$ given $\sigma^{2}$ and $\tau_{k}^{2}$ is defined by

$$
f_{\beta_{k} \mid \sigma^{2}, z_{k}=1}=\frac{1}{\sqrt{2 \pi\left(\sigma^{2} \tau_{k}^{2}\right)}} \exp \left\{-\frac{1}{2}\left[\frac{\beta_{k}^{2}}{\tau_{k}^{2} \sigma^{2}}\right]\right\} \text {. }
$$

Consequently, the prior distribution for $\beta_{k}$ conditioned to $\sigma^{2}$ and $Z_{k}=1$ is given by

$$
\begin{aligned}
f_{\beta_{k} \mid \sigma^{2}, z_{k}=1} & =\int_{0}^{\infty} \frac{\left(\frac{s_{1}^{2} \nu_{1}}{2}\right)^{\frac{\nu_{1}}{2}}}{\Gamma\left(\frac{\nu_{1}}{2}\right)} \exp \left\{-\frac{1}{2}\left[\frac{s_{1}^{2} \nu_{1}}{\tau_{k}^{2}}+\frac{\beta_{k}^{2}}{\tau_{k}^{2} \sigma^{2}}\right]\right\} \frac{1}{\tau_{k}^{2}\left(1+\frac{\nu_{1}}{2}\right)} d \tau_{k}^{2} \\
& \propto \int_{0}^{\infty} \exp \left\{-\frac{1}{2}\left[\frac{s_{1}^{2} \nu_{1}}{\tau_{k}^{2}}+\frac{\beta_{k}^{2}}{\tau_{k}^{2} \sigma^{2}}\right]\right\} \frac{1}{\tau_{k}^{2}\left(1+\frac{\nu_{1}}{2}\right)} d \tau_{k}^{2}
\end{aligned}
$$

To solve the integrate written in 16 we have to make the change of variable 


$$
u=\frac{1}{2 \tau_{k}^{2}}\left(\frac{\nu_{1} s_{1}^{2} \sigma^{2}+\beta_{k}^{2}}{\sigma^{2}}\right)
$$

and find $d \tau_{k}^{2}$ in terms of $u$ and $d u$. It is implies

$$
\tau_{k}^{2}=\frac{1}{2 u}\left(\frac{\nu_{1} s_{1}^{2} \sigma^{2}+\beta_{k}^{2}}{\sigma^{2}}\right)
$$

and

$$
d \tau_{k}^{2}=-\frac{1}{2} u^{-2}\left(\frac{\nu_{1} s_{1}^{2} \sigma^{2}+\beta_{k}^{2}}{\sigma^{2}}\right) d u
$$

Substituing both (17) and (18) in 16 we have

$$
\begin{aligned}
f_{\beta_{k} \mid \sigma^{2}, z_{k}=1} & \propto \int_{0}^{\infty}\left(\frac{\nu_{1} s_{1}^{2} \sigma^{2}+\beta_{k}^{2}}{\sigma^{2}}\right)^{\left(-\frac{\nu_{1}+1}{2}\right)} u^{\frac{\nu_{1}+1}{2}-1} \exp \{-u\} d u \\
& \propto\left(\frac{\nu_{1} s_{1}^{2} \sigma^{2}+\beta_{k}^{2}}{\sigma^{2}}\right)^{\left(-\frac{\nu_{1}+1}{2}\right)} \int_{0}^{\infty} u^{\frac{\nu_{1}+1}{2}-1} \exp \{-u\} d u \\
& \propto\left(\frac{\nu_{1} s_{1}^{2} \sigma^{2}+\beta_{k}^{2}}{\sigma^{2}}\right)^{\left(-\frac{\nu_{1}+1}{2}\right)} \Gamma\left(\frac{\nu_{1}+1}{2}\right) \\
& \propto\left(\nu_{1} s_{1}^{2} \sigma^{2}+\beta_{k}^{2}\right)^{\left(-\frac{\nu_{1}+1}{2}\right)} \Gamma\left(\frac{\nu_{1}+1}{2}\right) \\
& \propto\left(1+\frac{\beta_{k}^{2}}{\nu_{1} s_{1}^{2} \sigma^{2}}\right)^{\left(-\frac{\nu_{1}+1}{2}\right)} \\
& \propto\left(1+\frac{\beta_{k}^{2}}{\nu_{1} \tilde{s}_{1 \sigma^{2}}}\right)^{\left(-\frac{\nu_{1}+1}{2}\right)}
\end{aligned}
$$

showing that (19) is a kernel of scaled t distribution [24], [17] with degree of freedom $\nu_{1}{ }_{157}$ and scale parameter $\tilde{s}_{1 \sigma^{2}}=s_{1}^{2} \sigma^{2}$.

Likewise, for $Z_{k}=0$, we have

$$
\beta_{k} \mid \sigma^{2}, z_{k}=0 \sim \operatorname{Scaled} \mathrm{t}\left(0, \nu_{2}, \tilde{s}_{2 \sigma^{2}}\right)
$$

where $\tilde{s}_{2 \sigma^{2}}=s_{2}^{2} \sigma^{2}$.

Consequently,

$$
\left\{\begin{array}{lll}
\beta_{k} \mid \sigma^{2}, Z_{k}=1, \sim \operatorname{Scaled}-\mathrm{t}\left(\nu_{1}, \tilde{s}_{1 \sigma^{2}}\right) & \text { with probability } & \pi \\
\beta_{k} \mid \sigma^{2}, Z_{k}=0, \sim \operatorname{Scaled}-\mathrm{t}\left(\nu_{2}, \tilde{s}_{2 \sigma^{2}}\right) & \text { with probability } & (1-\pi)
\end{array}\right.
$$

showing that for $\left(\nu_{1}=\nu_{2}\right)$ and $\left(s_{1}^{2}=s_{2}^{2}\right)$, the prior distribution for each $\beta_{k}$ of bayes $\mathrm{H}$ model is equivalent the prior distribuition of bayes A model. Furthermore, for $s_{1}^{2}$ or $s_{2}^{2}$ tending to zero, the prior distribution for each $\beta_{k}$ is equivalent to bayes $\mathrm{B}$ model. There are other possibilities, for example, tending $s_{1}^{2}$ or $s_{2}^{2}$ to infinity, a mixture distribution of slab Gaussian and t-scaled distribuition is obtained as prior for each $\beta_{k}$.

\section{Results and Discussion}

In this study, we purposed a new hyperprior for bayesian regression model to predict complex trait. This model were applied in real and simulated datasets. 
Results from cross validation studies shows the prediction accuracy of Bayes $\mathrm{H}$ model is slight higher than Bayesian Ridge Regression in scenarios where dataset were generated from heterogeneous stock mice population and quite higher for dataset simulated from random mating. Hence, the type population, consequently, the strength of linkage disequilibrium is more influential in the prediction than number of QTLs (2). However, comparing two datasets generated from random mating, the number of QTLs caused a increase of prediction accuracy in Bayes $\mathrm{H}$ model. It was not observed difference in the prediction accuracy of Bayes $\mathrm{H}$ when were used different hyperpriors.
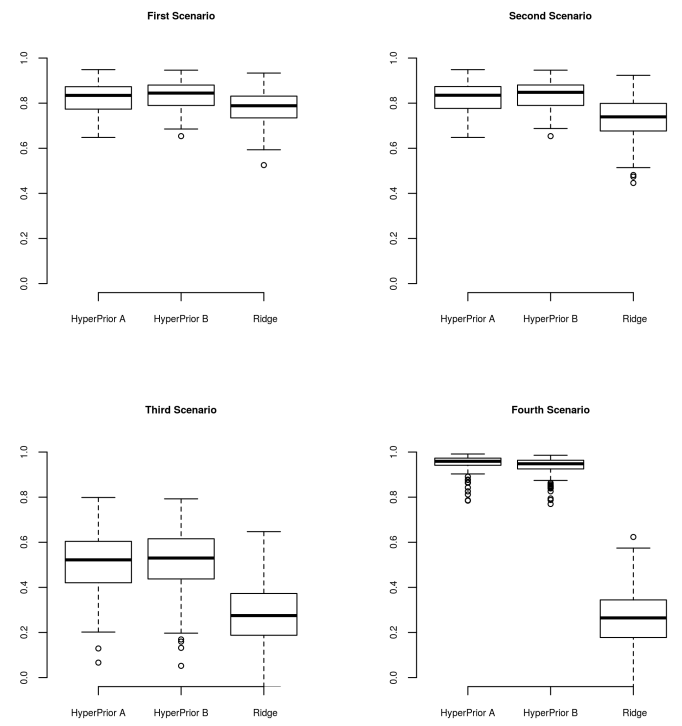

Fig 2. Evaluation of accuracy performance of Bayes $\mathbf{H}$ model using $\mathbf{5}$ fold cross validation. Box plot of Pearson's correlation distribution between observed and predicted values for each simulated scenario.

Figure (3) shows population structure inferred from top two eigenvectors obtained from correlation matrix of mice dataset. Bayesian information criterion indicates the best model is Gaussian mixture with 8 components. Thus, we can infer the presence of eight subpopulations which are the same number of founders of heterogeneous stock mice population 3. The scatterplot of top two eigenvector estimated from to genomic relationship matrix shows the clusters 3. Prediction accuracy of BMI was compared between differents hyperpriors of Bayesian Regression models in the heterogeneous stock mice population. In order to make the comparison, 5 fold cross validation was used. Box plot of Pearson's correlation distribution between observed and predicted values reveals moderate to high accuracy for all models 4. Moreover, the Bayesian ridge regression presented slight higher correlation in regarded to variable selecion model (hyperprior B) and quite higher correlation than model with hyperprior A.

A possible explanation of the fact that Bayes $\mathrm{H}$ model outperformed Bayes Ridge Regression only in a simulated dataset is the genetic architecture of the trait BMI. In the simulated data, the QTLs were the unique source of variation considered.

Furthermore, the number of QTLs used in the simulations were at most moderate (50). Therefore, models take into consideration that markers have different variances depending on their effects normally predict better than Bayesian Ridge Regression 10, 15. Using fat percentage dataset in a dairy cattle population, which a single gene explains $50 \%$ of the genetic variation, Verbyla et. al 26] reported that predictions from fitting of Bayes $\mathrm{Cp}$ have more accuracy than predictions obtained by 


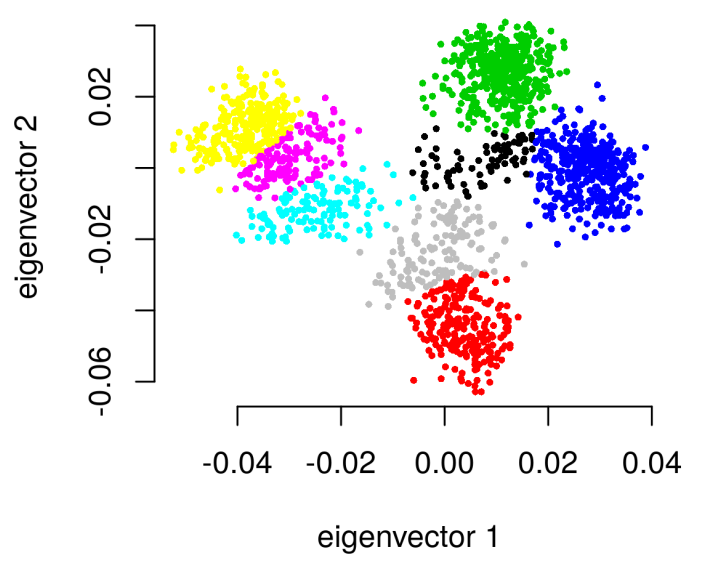

Fig 3. Population structure of heterogeneous stock mice.

RR BLUP. Moreover, Rezende et. al. [27 analyzed data from 17 traits measured in Pinus taeda population comprised of 951 individuals genotyped with 4853 SNPs. They concluded that for trait controlled by few genes, Fusiform rust for example, the models as Bayes A, Bayes Cp had higher predict ability in comparison to RR BLUP. On the other hand, BMI trait is considered a complex trait controlled by a large number of genes 25]. Thus, it is expected the RR BLUP would have a good predictive performance because this model considers homogeneous shrinkage of marker effects. The hypothesis is supported when we considered that genetic architecture of trait can be described by infitesimal model. However, we should have caution with these arguments, Gianola showed heuristically that Bayesian Ridge Regression or RR BLUP does not shrinkage the marker effects the same manner, the best linear unbiased predictor is sample size and allele frequency dependent 17, 18. Here we would like to speculate another hypothesis about the reason of good predictive performance of the Bayesian Ridge Regression in the real mice dataset. In the real dataset there are many source of genetic variation besides QTLS, such as: background genetic, linkage

disequilibrium, epistasis effects and etc. Consequently, the linear model declared in all Bayesian model is not true. Hence, the idea to select the markers that contribute the phenotypic variation does not work well. And this case, the prediction provided by RR BLUP or Bayesian Ridge Regression would be a better approximation.

Table 3. Gaussian mixture model selection using Bayesian Information Criterion

\begin{tabular}{cc}
\hline Modelo & BIC \\
\hline 1 & 16888.60 \\
2 & 18724.63 \\
3 & 19643.02 \\
4 & 19906.28 \\
5 & 20030.32 \\
6 & 20199.56 \\
7 & 20253.73 \\
8 & 20288.74 \\
\hline
\end{tabular}




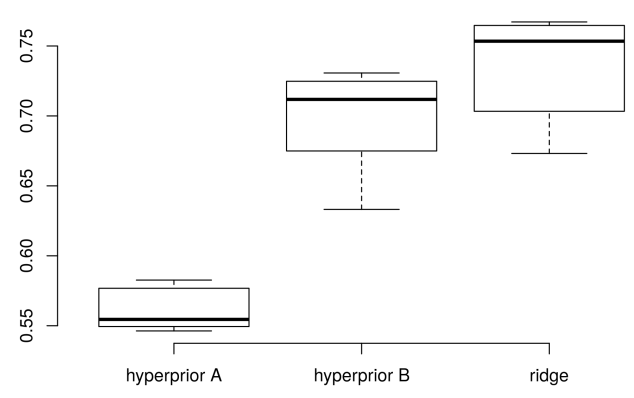

Fig 4. Evaluation of accuracy performance of Bayes $\mathbf{H}$ model using $\mathbf{5}$ fold cross validation. Box plot of Pearson's correlation distribution between observed and predicted values for mice dataset .

\section{References}

1. de los Campos G, Hickey JM, Pong-Wong R, Daetwyler HD, Calus MPL. Whole-Genome Regression and Prediction Methods Applied to Plant and Animal Breeding. Genetics. 2013;193(1):327-345. doi:10.1534/genetics.112.143313.

2. Hoerl AE, Kennard RW. Ridge Regression: Biased Estimation for Nonorthogonal Problems. Technometrics. 1970;12(1):55-67. doi:10.1080/00401706.1970.10488634.

3. Tibishirani R. Regression Shrinkage and Selection via the Lasso. Journal Royal Statistical Society B. 1996;58(1):267-288. doi:10.2307/2346178.

4. Frank IE, Friedman JH. A Statistical View of Some Chemometrics Regression Tools. Technometrics. 1996;35(2):109-135. doi:10.2307/1269656.

5. Fan J, Li R. Variable Selection via Nonconcave Penalized Likelihood and its Oracle Properties. Journal of the American Statistical Association. 2001;96(456):1348-1360. doi:10.1198/016214501753382273.

6. Fan J, Lv J. A Selective Overview of Variable Selection in High Dimensional Feature Space. Statistica Sinica. 2010;20(01):101-148.

7. George EI, McCulloch RE. Variable Selection via Gibbs Sampling. Journal of the American Statistical Association. 1993;88(423):881-889. doi:10.1080/01621459.1993.10476353.

8. Park T, Casella G. The Bayesian Lasso. Journal of the American Statistical Association. 2008;103(482):681-685. doi:http://dx.doi.org/10.1198/016214508000000337.

9. Heffner EL, Sorrells ME, Jannink JL. Genomic selection for crop improvement. Crop Science. 2009;49(1):1-12. doi:doi:10.2135/cropsci2008.08.0512.

10. Meuwissen THE, Hayes BJ, Goddard ME. Prediction of Total Genetic Value Using Genome-Wide Dense Marker Maps. Genetics. 2001;157(4):1819-1829.

11. Bernardo R. Breeding for Quantitative Traits in Plants 2nd Edition. Woddbury: Stemma Press; 1994. 
12. Lateef DD. DNA Marker Technologies in Plants and Applications for Crop Improvements. Journal of Biosciences and Medicines. 2015;3(5):7-18. doi:10.4236/jbm.2015.35002.

13. Hallauer AR, Carena MJ, Miranda Filho JB. Quantitative Genetics in Maize Breeding (Handbook of Plant Breeding, Vol. 6). New York: Springer; 2010.

14. Piepho HP. Ridge regression and extensions for genomewide selection in maize. Crop Science. 2009;49(4):1165-1179. doi:10.2135/cropsci2008.10.0595.

15. Habier D, Fernando RL, Kizilkaya K, Garrick DJ. Extension of the bayesian alphabet for genomic selection. BMC Bioinformatics. 2011;186(12):1-12. doi:10.1186/1471-2105-12-186.

16. Fisher, R. A. The correlation between relatives on the supposition of Mendelian inheritance. Trans. R. Soc. 1918.52:34.

17. Gianola D, de los Campos G, Hill WG, Manfredi E, Fernando R. Additive Genetic Variability and the Bayesian Alphabet. Genetics. 2009;183(1):347-363. doi:10.1534/genetics.109.103952.

18. Gianola D. Priors in whole-genome regression: The Bayesian alphabet returns. Genetics. 2013;90(3):525-540. doi:10.1534/genetics.113.151753.

19. Perez P, de los Campos G. Genome-wide regression and prediction with the BGLR statistical package. Genetics. 2014;3(2):483-495. doi:10.1534/genetics.114.164442.

20. Gilks WR, Richardson S, Spiegelhalter D. Markov Chain Monte Carlo in Practice. London: Chapman \& Hall; 1996.

21. Gelman A, Carlin JB, Stern HS, Dunson DB, Vehtari A, Rubin DB. Bayesian Data Analysis, 3rd ed. London: Chapman \& Hall; 2004.

22. de los Campos G, Naya H, Gianola D, Crossa J, Legarra A, Manfredi E, et al. Predicting Quantitative Traits With Regression Models for Dense Molecular Markers and Pedigree. Genetics. 2009;182(1):375-385. doi:10.1534/genetics.109.101501.

23. R: A Language and Environment for Statistical Computing; 2014. Available from: http://www.R-project.org/.

24. Box GEP, Tiao GC. Bayesian Inference in Statistical Analysis. New York: John Wiley and Sons, Inc; 1992.

25. Legarra A, Robert-Granié C, Manfredi E, Elsen JM. Performance of Genomic Selection in Mice. Genetics. 2008;180(01):611-618. doi:10.1534/genetics.108.088575.

26. Verbyla KL et. al. Accuracy of genomic selection using stochastic search variable selection in Australian Holstein Friesian dairy cattle. Genetic Research. 2009;91(5):307-11. doi: 10.1017/S0016672309990243.

27. Rezende MRF, et. al. Accuracy of Genomic Selection Methods in a Standard Data Set of Loblolly Pine (Pinus taeda L.) Genetics. 2012;190:1503-1510. doi: 10.1534/genetics.111.137026. 
28. Valdar W, Solberg LC, Gauguier D, Cookson WO, Rawlins JNP, Mott R, et al. Genetic and Environmental Effects on Complex Traits in Mice. Genetics. 2006;174(02):959-984. doi:10.1534/genetics.106.060004.

29. Fraley C, Raftery AE, Murphy TB, Scrucca L. mclust Version 4 for R: Normal 289 Mixture Modeling for Model-Based Clustering, Classification, and Density Estimation; 2012.

30. Fraley C, Raftery AE. Model-based Clustering, Discriminant Analysis and Density Estimation. Journal of the American Statistical Association. 2002;97(458):611-631. 